\title{
Structure and properties of the modified diffusion nitride-oxide surface layer
}

\author{
Kholikul Eshkabilov", Sherzod Berdiyev \\ Karshi Engineering Economic Institute, Karshi, Uzbekistan
}

\begin{abstract}
To combine the processes of gas nitriding and oxidation in water vapor, the effects of the atmospheric nitrogen potential in dissociated ammonia on the formation of surface diffusion nitride phases are studied. Modification of the surface nitride layer with oxygen was carried out by oxidation of the nitride zone at the second stage of obtaining an oxycarbonitride layer.

The corrosion and wear-resistant properties of the nitride-oxide layer $t$ of the lowest nitride layer are investigated, depending on the phase changes in the nitride layer after oxidation. The distributions of the elements in the nitride-oxide layer are determined, and the nature of the formation of the modified surface layer under the combination of gas nitriding with subsequent oxidation in water vapor is established.
\end{abstract}

\section{Introduction}

Currently, increasing the durability and reliability of machine parts and equipment through the use of modern methods of surface hardening, in particular, a combination of chemical and thermal processing methods is achieved by creating modified diffusion surface coatings in their most critical working units and parts. At the same time, the obtained structural materials with predetermined guaranteed properties, taking into account the specific operating conditions, should ensure the implementation of new surface physical-mechanical and physical-mechanical properties that arise during operation [1-4].

Nitriding and its varieties are among the most effective and widespread methods of surface modification of steels and alloys, increasing the corrosion resistance, wear resistance, and endurance limit of various machine parts and mechanisms. Various types of nitriding processes and combinations of nitriding with other surface hardening methods are widely used in the world of mechanical engineering [5-11].

One of the many possibilities of combining nitriding with other saturation methods is the creation of a controlled diffusion modified nitride layer with the formation of various structural and phase components on the hardened surface during processing. The nitride, carbonitride and oxycarbonitride zones, and the internal nitriding zones obtained after the processes of combining nitriding with other methods, are responsible for such performance characteristics as corrosion resistance, surface hardness and wear resistance [12-15].

The structure and phases of the nitride layer and the surface oxide layer obtained on the

*Corresponding author: kholik@rambler.ru 
surface of steels and alloys after the combined process of gas nitriding and superheated steam oxidation depend on various factors which are the nitrogen potential, temperature and saturation pressure. In the process of nitrooxidation, with a change in one or another of the above factors in the corresponding stages of saturation with nitrogen and oxygen, a nitride oxide layer can be formed on the surface of the hardened product with properties for specific operating conditions [16-18].

Predicting the structure, phase and properties of the nitride-oxide layer obtained by lowtemperature gas nitriding and subsequent oxidation in water vapor of metal products is important for optimizing the combined nitriding process with subsequent oxidation.

\section{Methods}

According to the technological sequence, the process of gas nitriding with subsequent oxidation is carried out according to the scheme of the combined process: nitriding in dissociated ammonia at the first stage of saturation and followed by oxidation in water vapor at the second stage.

Steel 45 in the delivery state was used as materials to study the combined process, which consists of nitriding followed by oxidation in water vapor.

Metallographic analysis of the treated steel samples was carried out on transverse sections using a Neophot-21 light microscope in the magnification range of 500-1000.

The nitriding process was investigated at higher and lower eutectoid temperatures for the "Fe-N" system and the second-stage oxidation in water vapor at higher and lower eutectoid temperatures for the "Fe-O" system».

Qualitative phase X-ray diffraction analysis of the diffusion nitride and nitride-oxide layers was studied using the Dron-3 diffractometer using filtered cobalt and iron $\mathrm{K}_{\alpha}-$ radiation [19-20].

The overall corrosion resistance of the treated samples was studied in a $3 \% \mathrm{NaCl}$ solution.

The distribution of elements in the layer depth was determined by Jelektronnoj spectroscopy on an electronic spectrometer. The microstructure of the layer obtained various options hardening treatment and was examined using the scanning electron scanning microscope JSM-25 "JEOL».

\section{Results and Discussions}

During nitriding, a nitride and carbonitride diffusion layer is formed, and the formation of a carbonitride layer occurs due to the carbon matrix of the steel. In the process of gas nitriding, the predominant diffusion of nitrogen occurs, and the structure and phase composition of the diffusion nitride layer is determined by the state diagram of the "ironnitrogen" system. On the surface of the processed materials, the formation of the phase composition and structure of the nitrided layer, depending on the nitrogen potential and the composition of the saturating medium, occurs with the formation of an internal nitriding zone and a further increase in the nitrogen concentration on the surface leads to the development of the $\varepsilon^{+} \gamma^{\prime}$-phase. To achieve maximum concentration of atomic nitrogen formed at the moment of decomposition of ammonia dissociation, which is accompanied by the release of atomic nitrogen in the active state, which soon turns into a molecular state and loses its activity:

$$
2 \cdot \mathrm{NH}_{3} \rightarrow 2 \cdot \mathrm{N}+6 \cdot \mathrm{H} \rightarrow \mathrm{N}_{2}+3 \cdot \mathrm{H}_{2}
$$

Of all the known saturating media, the most dynamic are gas atmospheres, which allow 
us to obtain any structural sets and a different range of depths of the diffusion layers. In all cases of gas nitriding, ammonia is the base for creating a saturating atmosphere. Intensive nitrogen diffusion to the surface occurs only when the dissociation of ammonia occurs in the immediate vicinity of atomic nitrogen from the nitrided surface.

During the saturation process, a certain nitrogen potential is formed, and during the process, the nitrogen potential is balanced. According to the relation (1), a certain part of atomic nitrogen is diffused to the metal surface, which is formed after a catalytic reaction, as a result of which MeT metal nitrides are formed. Moreover, on the surface of metals or alloys, higher metal nitrides first develop to reduce the concentration of nitrogen at a depth of the saturated surface.

In short-term gas nitriding, the nitriding process first occurs with the formation of a nitride layer. In all cases, the formation of the nitride layer and the internal nitriding zone in pure iron occurs following the "Fe-N" diagram, and on carbon steels occurs following the "Fe-N-C" diagram».

By maintaining the nitrogen potential at the level of nitrogen solubility in one or another phase, it is possible to obtain a layer consisting of an $\alpha$-solid solution, iron nitrides and alloying elements, and to form a $\gamma^{\prime}$-phase (low nitride) or a $\varepsilon^{\prime}$-phase with a low nitrogen content on the surface.

As the value of the nitrogen potential increases, the thickness of the resulting diffusion nitride layer increases. Under the same conditions obtained, the samples were examined by X-ray diffraction analysis (Figure 1). Analysis of changes in diffraction peaks of the reflection phases shows that the increase of the nitrogen potential in the nitride layer increases the proportion of R-phase, and neskatoties $\gamma^{\prime}$-phase and $\alpha$-phase (zone of internal nitriding) is reduced. The optimal nitride layer by phase composition can be obtained at the values of the nitrogen potential: $2<\pi_{N}<3$.

With increasing nitrogen capacity $\pi_{N}>1$ receives on the surface of well-developed nitride layer consisting of $\varepsilon^{\prime}$ - and $\varepsilon^{\prime}+\gamma^{\prime}$-phase with increasing the nitrogen potential to $\pi_{N}$ $<6$ proportion of $\gamma^{\prime}$-phase in nitride layer decreases, and $\varepsilon^{\prime}$-phase is increased.

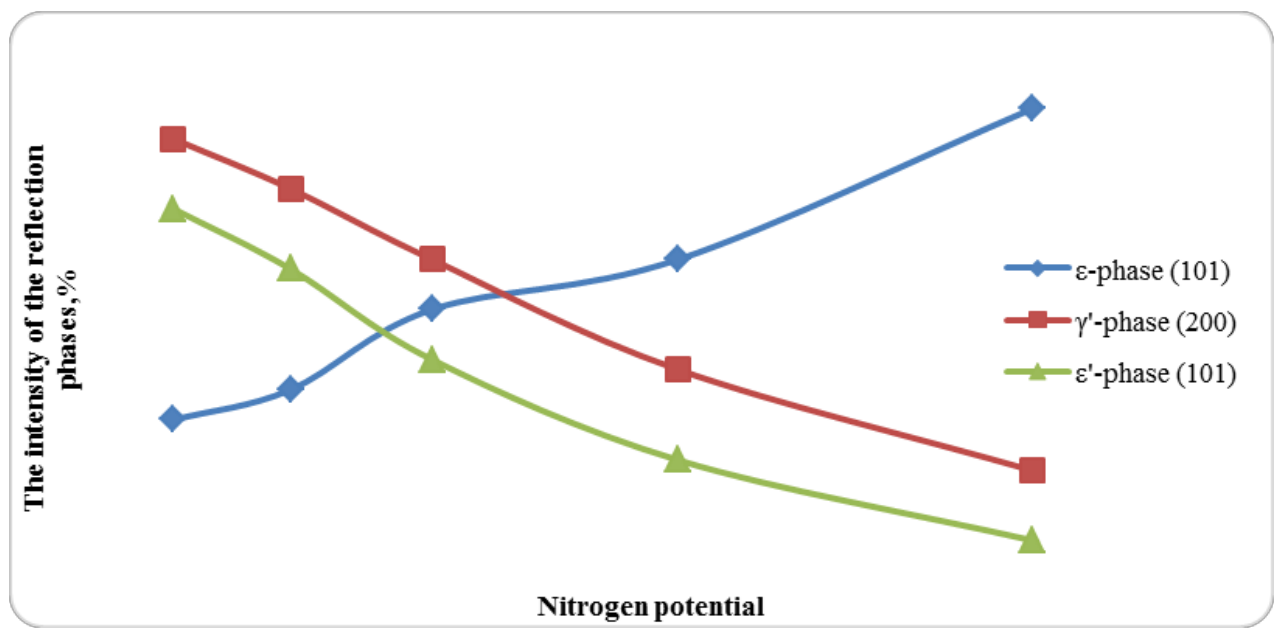

Temperature $-580^{\circ} \mathrm{C}$, duration -3 hours.

Fig.1. Change in the diffraction maxima as a function of the nitrogen potential of the atmosphere during nitriding,

The increase in the thickness of the nitride layer leads to the hindrance of diffusion of 
nitrogen through the nitride layer, if necessary, increase the duration of nitriding, as well as with increasing nitrogen potential $\pi_{N}>5$ due to the increase in the share of high nitride layer reveals the fragility of the layer and the porosity $\varepsilon$-phase.

To obtain a pore-free low-nitrogen diffusion nitride layer for further oxidation, it is desirable to obtain a low-nitrogen nitride layer consisting of a low-nitrogen $\varepsilon$-phase or a mixture of $\left(\varepsilon+\gamma^{\prime}\right)$-phases. The nitride layer formed when saturated with nitrogen surface in the active period has a high concentration of nitrogen in the second stage of saturation under low nitrogen potential to achieve equilibrium can dissociate in two areas: the process of diffusion of nitrogen from the nitride layer deep into the metal and return back into the environment.

In the process of nitrogen diffusion from the nitride layer into the metal, the atmosphere in the furnace does not have a direct effect; in this case, the determining factors are the temperature and composition of the processed material (the presence of carbon in the matrix).

The return process of atomic nitrogen from the nitride layer back into the environment (air furnace), you can stop by the establishment of the furnace atmosphere by creating the necessary condition for equilibrium nitrogen potential with a decrease in the flow rates of ammonia in an oven or create a barrier surface layer hindering the process of diazotation.

The formation of the e ' - phase occurs only when nitriding steel and alloys in the presence of carbon in their matrix and has a carbonitride character. Therefore, a noticeable formation of this type of nitride is characteristic only when nitriding carbon steel and alloys.

To obtain a complete picture of the change in the nitride layer during oxidation, samples from steel 45 were studied. The samples were processed in an atmosphere of pure ammonia at nitrogen potential values in the range $2<\pi_{N}<3$, at a temperature of $580^{\circ} \mathrm{C}$-below the eutectoid temperature for the "Fe-N" system, and some of the samples, without removing them from the furnace, were oxidized in water vapor at a temperature of $550^{\circ} \mathrm{C}$ - below the eutectoid temperature for the "Fe-O" system. Microslips were made from the processed samples, and the distribution of elements over the depth of the oxide and nitride layers was studied.

The oxidation at the second stage was carried out in two versions: in pure water vapors in vapors of a 5\% solution of complexions (in particular, ODEF) in water. On the surface of the samples treated in water vapor during cooling without isothermal exposure, microcracks formed in the oxide layer. Studies of surface morphology and X-ray diffraction analyses have shown that the occurrence of micro cracks in the surface oxide layer is due to the formation $\mathrm{FeO}$ in the oxide layer during the oxidation period, even at a temperature below the eutectoid for the "Fe-O" system (Figure 2, a).

When the nitride layer is oxidized in water vapor with the addition of complexions, no micro cracks are formed, and the appearance of $\mathrm{FeO}$ reflexes in diffractograms is not detected, the surface becomes smoother (Figure 2, b).

When the nitride layer is oxidized in water vapor with the addition of complexions, no micro cracks are formed, and the appearance of $\mathrm{FeO}$ reflexes in diffractograms is not detected, the surface becomes smoother (Figure 2, b). 


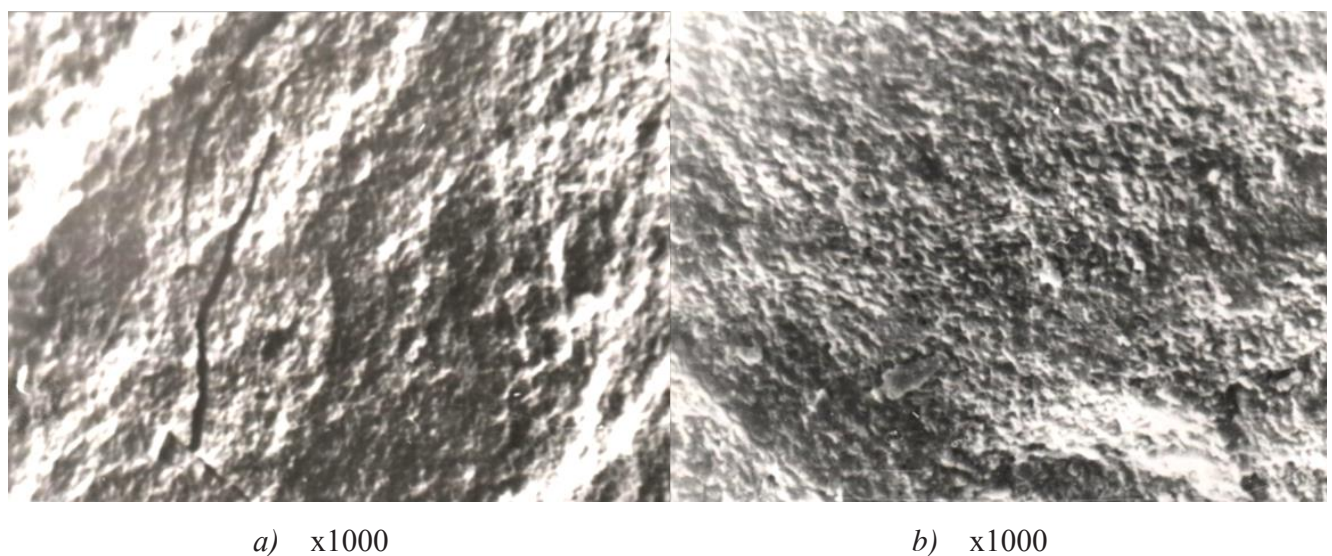

Nitriding- $580^{\circ} \mathrm{C}, 3 \mathrm{~h}$; oxidation- $550^{\circ} \mathrm{C}, 1 \mathrm{~h}$ : a - in water vapor; $\mathrm{b}$ - in a solution of EDF in water.

Fig. 2. Morphology of the surface of the nitride-oxide layer on steel 45

The microstructures of the surface with a nitride-oxide layer of steel 45 after nitriding at the first stage at temperatures below the eutectoid for the "Fe-N" system $\left(591^{\circ} \mathrm{C}\right)$ and subsequent oxidation in complex-containing water vapor at a temperature below the eutectoid for the "Fe-O" system $\left(550^{\circ} \mathrm{C}\right)$ are shown in Figure 3.

The surface oxide layer is formed by the diazotation nitride layer. From a theoretical point of view, the concentration of diffusing nitrogen during oxidation should gradually change from the core to the metal surface over time. However, the oxidation process of formation of the oxide layer at the initial time occurs in the superficial part and achieving the required density $(1-3 \mu \mathrm{m})$ oxide layer acts as barring layer, and the diazotation nitride layer is terminated, and the growth nitride layer due to the high dissociation nitride layer.

During oxidation, the introduction of complexions into the saturating medium, although in small amounts, favorably leads to forming a surface oxide layer over the nitride layer, forming a uniform, dense thin barrier layer of oxide. The surface oxide layer, in its turn, acts as a barrier layer and prevents the de-nitriding process. Simultaneously, the higher nitride phases dissociate towards the internal nitriding zone with an increase in the thickness of the low-nitrogen nitride phase due to the high concentration of nitrogen in the nitride layer (Figure 3, a). The dissociation of the nitride phase is especially noticeable with an increase in the holding time during oxidation (Figure 3, b).

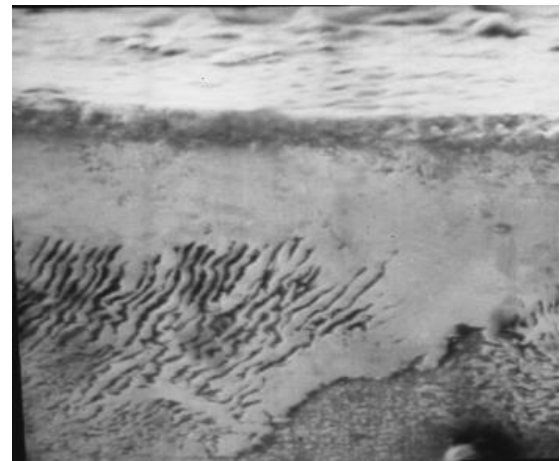

a) $x 1000$

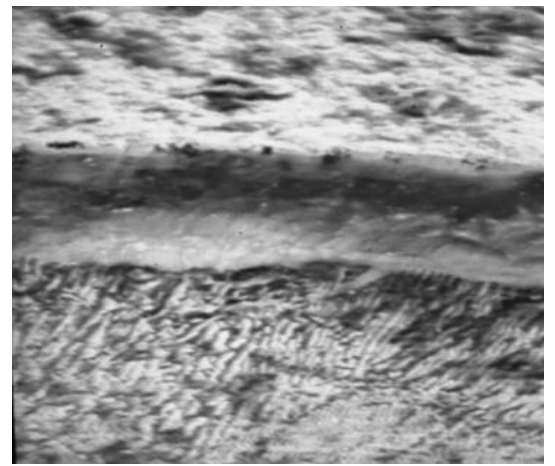

b) $\quad x 1000$

Nitriding $-580^{\circ} \mathrm{C}, 3$ hours: a is oxidation $-550^{\circ} \mathrm{C}, 1$ hour; $\mathrm{b}$ is oxidation- 2.5 hours.

Fig.3. Microstructure of the nitride - oxide layer on steel 45 
After oxidation, due to the diazotation of the samples, up to a certain depth, the nitrogen content is absent, and an increase in nitrogen is detected along with the nitride depth due to the diffusion of nitrogen in the nitride zone. When oxidation due to oxygen diffusion at the same time, diazotation and decarburization in the lower part of the nitride zone. An oxycarbonitride layer is formed on the surface.

The growth of the oxycarbonitride surface layer ( $\varepsilon^{\prime \prime}$-phase) occurs on the surface part of the nitride layer mainly due to the diffusion of oxygen from the atmosphere and nitrogen in the nitride layer, as well as carbon from the steel matrix. With an increase in the oxidation time, the thickness of the nitride layer decreases, which is accompanied by the dissociation of the high-nitrogen zone (Figure 4).

Recently, for surface hardening of parts working in a corrosive environment, for wear at low contact loads, a nitride - oxide layer consisting of a developed nitride zone and a thin surface oxide zone provides better wearability of the rubbing surfaces and corrosion resistance. Here, preference is given to the subsurface nitride zone, consisting of $\varepsilon$-phase and $\gamma^{\prime}$-phase, preferably a $\varepsilon$-phase with lower nitrogen content, having a carbonitride or oxycarbonitride character.

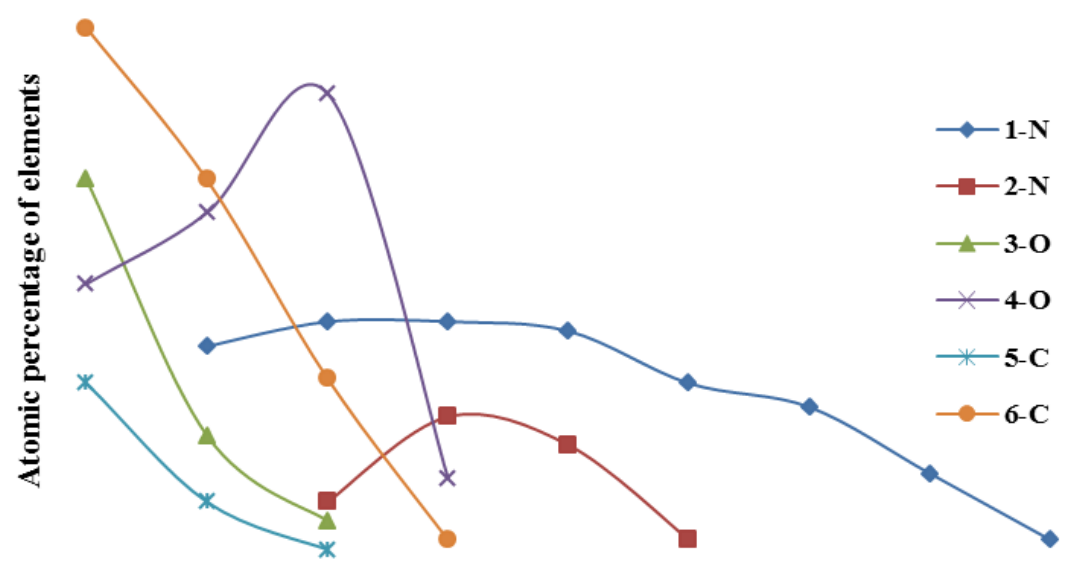

Distance from the surface, microns

Nitriding: $\mathrm{t}=580^{\circ} \mathrm{C}, \tau=3 \mathrm{~h}$; oxidation: $\mathrm{t}=550^{\circ} \mathrm{C}, \tau=1 \mathrm{~h}$ (lanes $\left.1,3,5\right)$ and $\tau=2.5 \mathrm{~h}$ (lanes $2,4,6$ ).

Fig.4. Distribution of elements in the nitride-oxide layer

For parts operating under fatigue conditions, at elevated temperatures, a nitride - oxide layer with a developed diffusion sub-layer is used, and for tools operating in the mode of dynamic wear and shock loads, an internal nitriding zone is formed on the surface without a brittle surface nitride layer.

Optimal corrosion-protective properties are achieved with an oxycarbonitride layer with a surface dense oxide zone consisting of magnetite $\left(\mathrm{Fe}_{3} \mathrm{O}_{4}\right)$ with a thickness of 1 to 3 microns. The change in the phase composition of the oxide zone with the formation of $\mathrm{FeO}$ and $\mathrm{Fe}_{2} \mathrm{O}_{3}$ oxides is accompanied by the loosening of the layer and leads to a deterioration in corrosion resistance.

The nitride zone with high surface characteristics has $\gamma^{\prime}$ - and $\varepsilon$-phases. Moreover, the $\varepsilon$ phase is nitrogen-depleted, and X-ray diffraction reveals two isomorphic phases: the isomorphic carbonitride phase, designated as the $\varepsilon^{\prime}$-phase with lattice parameters: $a=0.269$; 
$\mathrm{c}=0.436 \mathrm{~nm}$ and the carbonitride phase with lattice parameters: $\mathrm{a}=0.267 ; \mathrm{c}=0.436 \mathrm{~nm}[8$, 21].

The corrosion resistance of the nitride-oxide layer depends on the ratio of the $\gamma^{\prime}$ - and $\varepsilon$ phases in the nitride zone. Figure 5 shows the dependence of the time of occurrence of the first foci of corrosion in a $3 \%$ aqueous solution of table salt of steel 45 after nitrooxidation with water vapor additives. With an increase in the amount of the $y$ 'phase in the nitride zone to $75 \%$, the time until the first corrosion foci appear increases, reaching 910-960 hours at $75 \%$ of the $\gamma^{\prime}$-phase. A further increase in the amount of the $\gamma^{\prime}$-phase is accompanied by a decrease in corrosion resistance. The presence of an oxide layer has a positive effect on the corrosion properties of the nitride-oxide layer (Figure 5, line 1). It should be noted that in the experiments carried out, the thickness of the oxide zone was 1-5 microns.

In nitrooxidation, a composite layer consisting of almost a $\gamma^{\prime}$-phase can be obtained by a two-stage change in the nitrogen potential of the atmosphere at $560-580{ }^{0} \mathrm{C}$, and by preliminary dissociation of ammonia $\alpha=25 \%$ with the maintenance of dissociation of the ammonia mixture at saturation $\alpha=55-75 \%$ and a duration of $1.5-3$ hours.

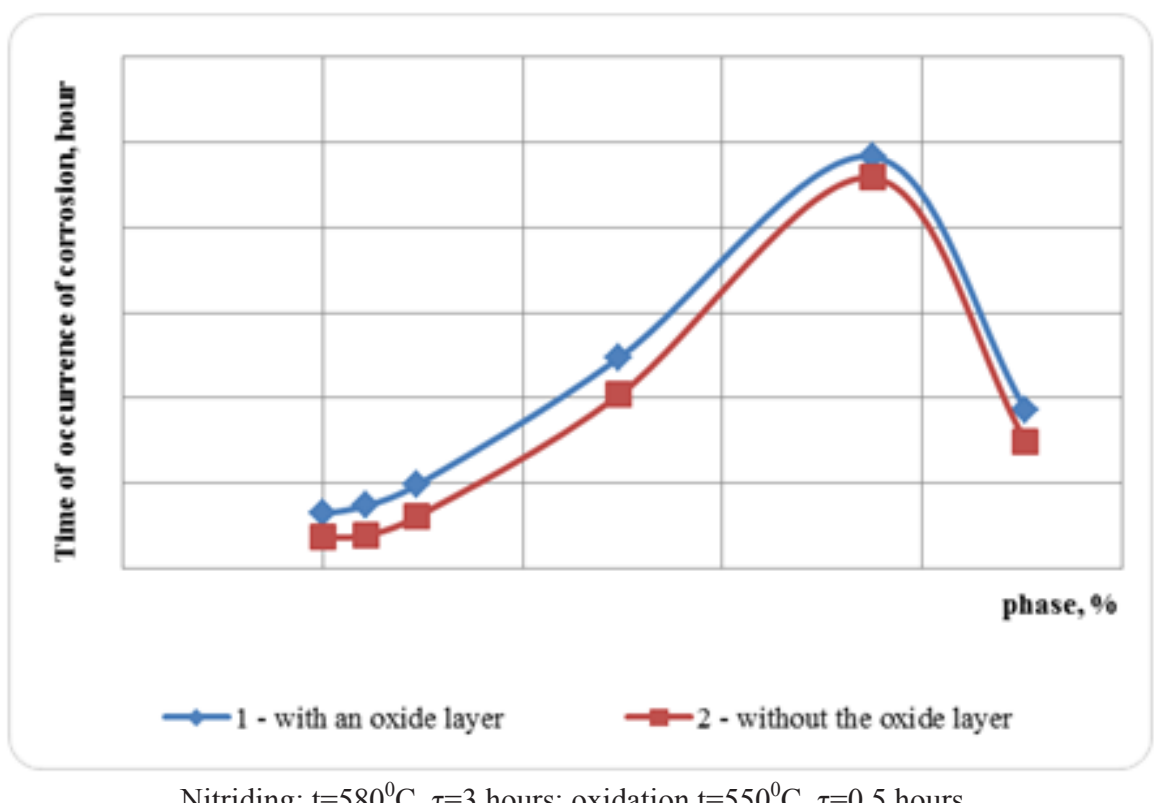

Nitriding: $\mathrm{t}=580^{\circ} \mathrm{C}, \tau=3$ hours; oxidation $\mathrm{t}=550^{\circ} \mathrm{C}, \tau=0.5$ hours.

Fig. 5. Influence of the concentration of the $\gamma^{\prime}$-phase in the nitride - oxide zone of steel 45 on the time of the appearance of the first foci of corrosion in $3 \% \mathrm{NaCl}$ solution

By subsequent oxidation of the $\gamma^{\prime}$-phase in water vapor at a temperature of $540-550^{\circ} \mathrm{C}$ for 0.5-1 hour, a uniform oxide layer with a thickness of 1-3 microns can be obtained on the surface. The resulting nitride-oxide layer has better corrosion properties. Under conditions of dry and boundary sliding friction, the wear resistance of 6-8 times greater than the nitrided layers obtained in an ammonia atmosphere at $580^{\circ} \mathrm{C}$ for 3 hours. With an increase in wear resistance, there is a decrease in the nitro oxide layer's coefficient than the nitride layer obtained by nitriding.

It is necessary to precisely match the parameters of the oxidation process at the second stage with the parameters of nitriding at the first stage. If they are not matched, an intermediate $\mathrm{Fe}_{\alpha}$-phase can form, which leads to surface cracking or peeling of the oxide layer. 


\section{Conclusions}

The results of the conducted studies have shown that:

- by setting the time and selecting the nitrogen potential of the saturating atmosphere during nitriding for its further oxidation, it is possible to obtain the necessary ratios of nitrogen phases in the nitride layer, which together have the necessary properties of the surface oxide layer;

- by adjusting a particular parameter of nitrooxidation at each stage of the process, it is possible to obtain a diffusive surface layer consisting of an oxide, nitride, carbonitride, and oxycarbonitride character;

- the nitride zone with high surface characteristics consists of the $\gamma^{\prime}\left(\mathrm{Fe}_{4} \mathrm{~N}\right)-$ and $\varepsilon^{\prime \prime}$ $\left(\mathrm{Fe}_{3} \mathrm{~N}\right)$ - phases. The corrosion resistance depends on the ratio of the $\gamma^{\prime}$ - and $\varepsilon^{\prime \prime}$-phases in the nitride zone. An increase in the amount of lower nitride ( $\gamma^{\prime}$-phase) in the nitride zone up to $75 \%$ is accompanied by an increase in corrosion resistance;

- the nitride-oxide layer, consisting of the $\gamma^{\prime}$-phase and the surface oxide $\mathrm{Fe}_{3} \mathrm{O}_{4}$, provides a positive gradient of properties, has good workability;

- in the process of water vapor oxidation of the nitride layer on structural steels, it is possible to obtain adjustable dense nitride-oxide layers responsible for certain specific operating conditions.

\section{References}

1. Lakhtin Y. M., Kogan Ya. D. Nitriding of steel, Mechanical engineering, p 256. (1976)

2. Lakhtin Yu. M., Kogan Ya. D. Structure and strength of nitrided alloys, M., Metallurgy, p176, (1982)

3. Lakhtin Yu.M., Kogan Ya.D., Shpis G.I.Byomer Z. Theory and technology of nitriding, M: Metallurgy. p. 320, (1991).

4. Rybakova J.I. M. Influence of the structural state of surface layers on the processes of friction and wear, Friction and wear. 8, (5). pp. 888-894, (1987)

5. Chatterjee-Fisher R., F.V. Eisell, R. Hoffman., Trans. from German Nitriding and carbonitriding, M.: Metallurgiya, p. 280, (1990)

6. Gerasimov S. A., Kuksenova L. I. Structure and wear resistance of nitrided steels. M.: Bauman Moscow State Technical University, p. 48. (2002)

7. Lakhtin Yu.M. Oxynitriding (Nitrooxidation), Metal science and heat treatment of metals, 9. pp .2-5, (1994)

8. Kogan Ya. Eshkabilov Kh. Oxynitriding metal products, Strengthening technologies and coatings. 6. pp 10-15. (2006)

9. Kogan Ya.D., Eshkabilov Kh.K. Combination technology for obtaining nitride-oxide coatings on structural steels, Scientific and Technical Conference on Complex Methods for Enhancing Reliability and Durability of Production Equipment Parts, Russian, Penza, pp 14. (1992)

10. David Pye. Practical Nitriding and Ferritic Nitrocarburizing, ASM Publication, p. 256, (2003)

11. Bogdanova N.V., Kochergin A.S., Kudryashov G.A. Research and development of an alternative to hard chrome plating technical process of hardening the pistons of brake cylinders of VAZ cars. Metal science and heat treatment of metals, 10. pp. 23-24. (2002)

12. Gladkova E.N. Theoretical foundations and technology of steam-thermal oxidation, Saratov, Saratov Polyt. Institute, p. 123

13. Lakhtin Yu.M., Arzamasov B.N. Chemical heat treatment of metals, M, Metallurgy, 
pp. 256. (1985).

14. Tikhomirov V.I. The rate of iron oxidation in water vapor and carbon dioxide at high temperatures, 95, pp. 305-309, Russia, (1994)

15. Samsonova G.V, Physical and mechanical properties of oxides, Directory, Moscow, Metallurgy, p. 472, (1976)

16. Margulova T.Kh. The use of chelators in heat power engineering. -M., Energoatomizdat, p. 37-43, (1986)

17. Kogan Ya.D., Eshkabilov Kh.K. Oxynitriding metal products, Strengthening technologies and coatings, 6 (18). pp. 10-16, Moscow, (2006)

18. Eshkabilov Kh. K., Berdiev Sh. A. Change in the structure and composition of the nitride layer during water vapor oxidation. Young Scientist, 14. M, pp. 204-207. (2016)

19. Eshkabilov Kh. K., Yurshev V. I. The University complex as a regional center of education, science and culture Nitrooxidative steel products, Materials of all-Russian scientific-methodical conference, Orenburg, January 23-25, pp. 787-793, ( 2020)

20. Gorelik S.S., Rastorguev A. N., Skakov Yu. L. x-Ray and electron-optical analysis. M.: Metallurgy, p 368. (1970)

21. Mirkin L. I. Handbook of $x$-Ray structural analysis of polycrystals, M.: MGU p 383, (1975)

22. Kh.K.Eshkabilov, Sh.A.Berdiyev, B.Kamolov. Hardening of cutting tools by combined gas nitriding method, VII International Scientific Conference "Integration, Partnership and Innovation in Construction Science and Education" Materials Science and Engineering, 1030, Tashkent, (2020) 